\title{
乌s \\ Linearization of the longitudinal phase space without higher harmonic field
}

\author{
Benno Zeitler, ${ }^{1, *}$ Klaus Floettmann, ${ }^{2}$ and Florian Grüner ${ }^{1}$ \\ ${ }^{1}$ Center for Free-Electron Laser Science and Department of Physics, \\ University of Hamburg, Luruper Chaussee 149, 22761 Hamburg, Germany \\ ${ }^{2}$ Deutsches Elektronen-Synchrotron DESY, Notkestraße 85, 22603 Hamburg, Germany
}

(Received 20 July 2015; published 30 December 2015)

\begin{abstract}
Accelerator applications like free-electron lasers, time-resolved electron diffraction, and advanced accelerator concepts like plasma acceleration desire bunches of ever shorter longitudinal extent. However, apart from space charge repulsion, the internal bunch structure and its development along the beam line can limit the achievable compression due to nonlinear phase space correlations. In order to improve such a limited longitudinal focus, a correction by properly linearizing the phase space is required. At large scale facilities like FLASH at DESY or the European XFEL, a higher harmonic cavity is installed for this purpose. In this paper, another method is described and evaluated: Expanding the beam after the electron source enables a higher order correction of the longitudinal focus by a subsequent accelerating cavity which is operated at the same frequency as the electron gun. The elaboration of this idea presented here is based on a ballistic bunching scheme, but can be extended to bunch compression based on magnetic chicanes. The core of this article is an analytic model describing this approach, which is verified by simulations, predicting possible bunch length below $1 \mathrm{fs}$ at low bunch charge. Minimizing the energy spread down to $\sigma_{E} / E<10^{-5}$ while keeping the bunch long is another interesting possibility, which finds applications, e.g., in time resolved transmission electron microscopy concepts.
\end{abstract}

DOI: 10.1103/PhysRevSTAB.18.120102

PACS numbers: 29.20.-c, 29.27.-a, 41.85.-p, 41.75.Fr

\section{INTRODUCTION}

Modern accelerators like free-electron lasers require ultrashort bunches on the femto-second scale. Apart from design considerations like a sufficient peak current, the bunch length ultimately determines the time resolution achievable with such light sources. The latter is also true for electron sources built to carry out time-resolved electron diffraction experiments, like, for example, the Relativistic Electron Gun for Atomic Exploration, REGAE [1,2], located at DESY in Hamburg.

To compress an electron bunch, a linearly correlated energy spread is impinged, making use of either subsequent time of flight differences based on the velocity differences of the particles (ballistic bunching, [3,4]) or on path length differences as caused by magnetic chicanes [5]. The compression is limited by two factors: space charge repulsion and nonlinear phase space correlations.

For example, at REGAE the bunches are compressed via a ballistic bunching scheme, leading to a longitudinal extent of the bunches down to $2 \mu \mathrm{m}$ according to simulations using the code ASTRA [6], corresponding to $7 \mathrm{fs}$. A further compression of the electron bunches down to even

\footnotetext{
*benno.zeitler@desy.de

Published by the American Physical Society under the terms of the Creative Commons Attribution 3.0 License. Further distribution of this work must maintain attribution to the author $(s)$ and the published article's title, journal citation, and DOI.
}

shorter length is limited by phase space correlations, originating from the curvature of the accelerating fields and nonlinear shifts of the particles within a drift: In an ideal focus, the phase space forms a straight upright particle distribution without correlated distortions. The longitudinal emittance, i.e., the product of bunch length and energy spread at the focus, is minimal in this case, limited only by uncorrelated contributions. Typically, however, the phase space forms rather a parabolic shape. Emittance and bunch length are hence enlarged due to correlated distortions.

One approach to eliminate the second and third order correlations is the usage of a decelerating higher harmonic rf field [7-9]. For example, an rf structure running at the triple frequency of the accelerating cavities is routinely operated at FLASH, DESY's XUV free-electron laser [10], and will also be employed at the European XFEL linac [11]. A simulation of REGAE with an added third harmonic cavity yields bunch lengths of 600 as [3].

The need for a different (shorter) rf wavelength in order to linearize the phase space curvature arises from the fact that the use of the same frequency would require to also apply the same amplitude-and, therefore, the bunch would be completely decelerated. This is, however, only true if there is no evolution of the bunch in between the accelerating and the linearizing cavity. Thus, another possibility to eliminate the nonlinearities is the acceleration in the gun far off-crest, referred to as stretcher mode in this article: The induced energy spread by such gun phase settings leads to a controlled expansion of the bunch in the 
(a)

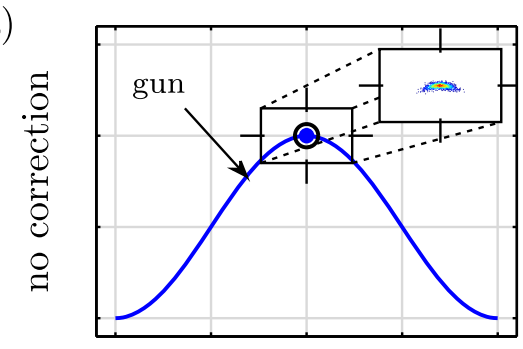

drift

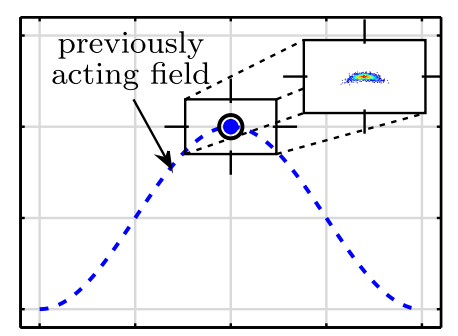

buncher

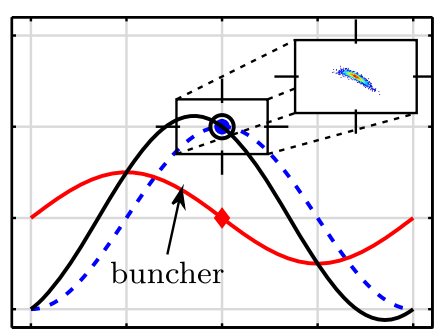

(b)

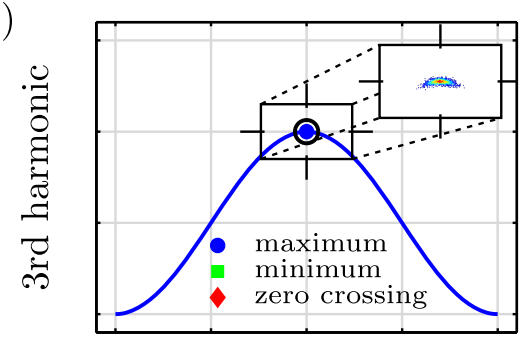

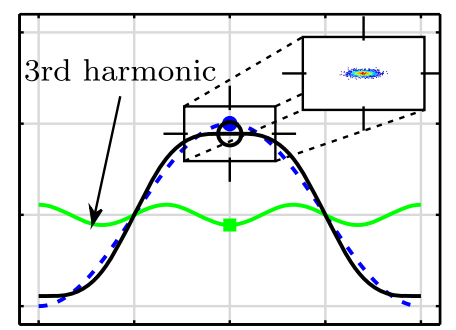

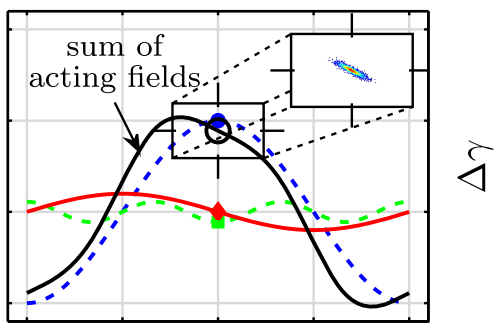

(c)
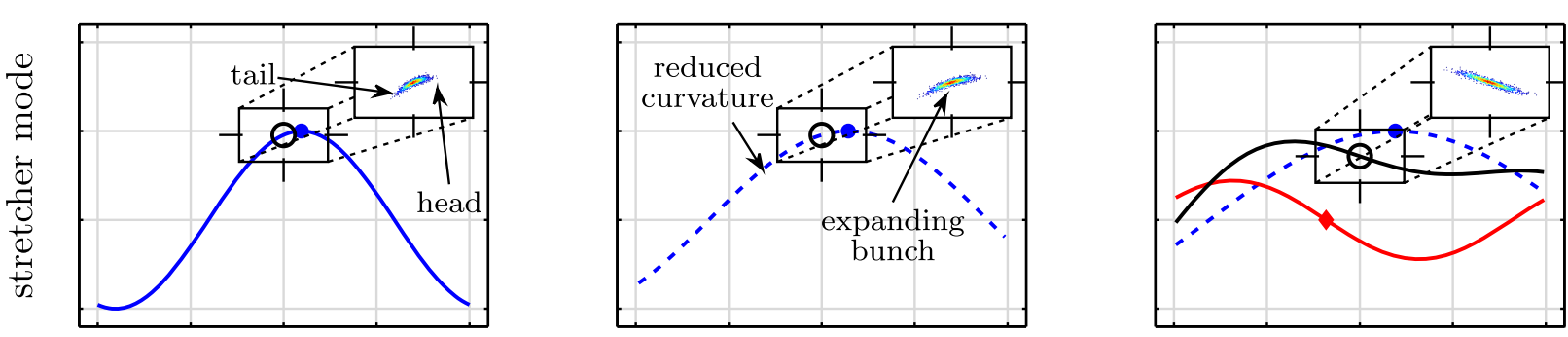

$\zeta$ (arb. units)

FIG. 1. The basic idea of the stretcher mode concept compared to an uncorrected bunch compression scheme and a third harmonic approach. The plots from left to right show the energy gain/loss, $\Delta \gamma$, in the respective cavity in the longitudinal phase space of the bunch. Previously acting fields are marked by dashed lines and the black line indicates the sum of all fields that acted up to the respective location. The small insets depict the shape of the bunch in the longitudinal phase space, resulting from an acceleration at the marked phase. Figures (a) depict the case without correction. The bunch acquires the curvature of the finite phase interval it covers in of the gun field-where for simplicity slippage has been neglected. The curved shape is transported and conserved during the drift. After the buncher cavity, a linear correlated energy spread has been imprinted on top by passing at the zero-crossing phase. However, the curvature is still present in the resulting bunching configuration, limiting the maximal achievable compression. In case (b), the introduction of a decelerating third harmonic cavity in the drift between gun and buncher flattens the phase space distributions, resulting in an almost purely linear $\zeta$-dependency of the energy distribution behind the buncher cavity. In (c), the stretcher mode, the bunch is accelerated off-crest in the gun, leading to a controlled expansion within the drift-and an accompanied reduction in the curvature due to the lengthening. Hence, it is possible to find a solution that linearizes the phase space by adding the negative (reduced) curvature in the buncher cavity, while simultaneously adding a linearly correlated energy spread, resulting in a bunch similar to the case of a third harmonic cavity. Please note, in this example only the action of the fields and the linear expansion for the off-crest case are considered. In the full picture higher order effects in the bunch kinetics also need to be explored, as will be seen in the course of this article.

subsequent drift, and consequently the phase space curvature and higher order correlations change as well. Hence, the subsequent linearizing cavity — which can in this case be simultaneously used for bunching - can be operated at the same frequency as the gun, but the field of the linearizer effectively acts like a higher harmonic structure, because the associated field curvature is larger than the expanded bunch nonlinearities. To illustrate the concept, a schematic comparison of the three cases is shown in Fig. 1.

The stretcher mode approach for ballistic bunching is developed and analyzed in the following article, which is structured as follows. In Sec. II, the mathematical formalism is derived, covering the nonlinear evolution of the phase space coordinates of the bunch particles based on the 


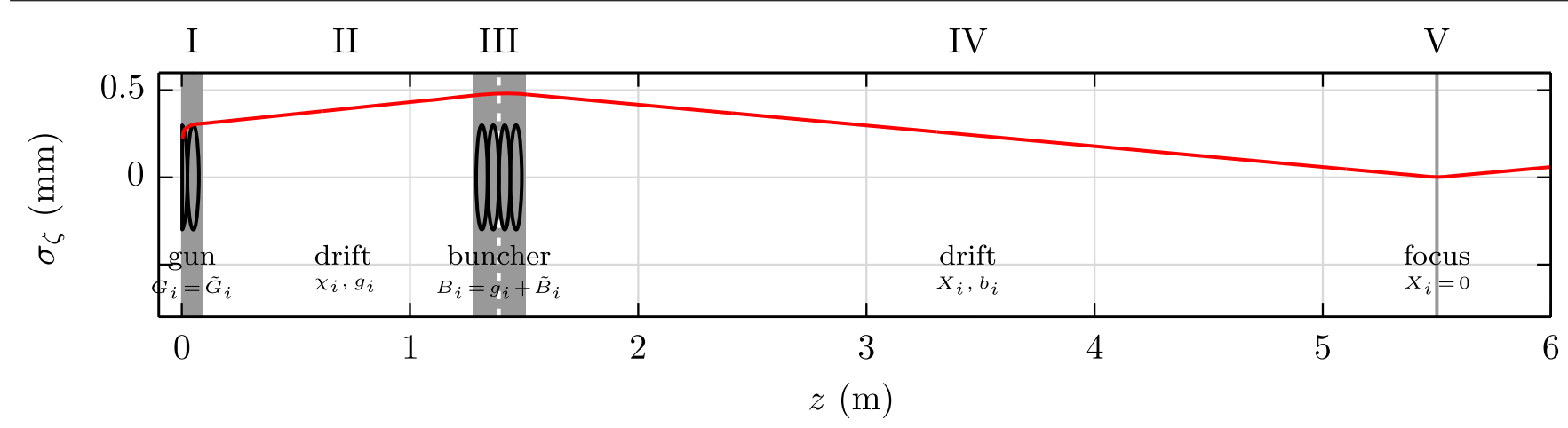

FIG. 2. Schematic of the cavity positions at REGAE and the drift spaces up to the longitudinal focus; an exemplary bunch length evolution is indicated by the red line. The electron gun is at (I), followed by a first drift section, (II), until the buncher cavity is reached at (III); the dashed, white line indicates the thin lens approximation of the buncher cavity. The second drift is marked by (IV) and delimited by the longitudinal focus, (V). The shaded zones will be kept for all drift plots throughout this article for illustration purposes. The parameters $\tilde{G}_{i}, G_{i}, g_{i}, \tilde{B}_{i}, B_{i}$ and $b_{i}$ will be used in the course of the article to describe the energy of the bunch as a polynomial in the respective zones, while $\chi_{i}$ and $X_{i}$ are the polynomial coefficients for the description of the spatial shift of particles in the longitudinal phase space of the bunch.

kinetics in a free drift, while cavities are treated as thin lenses, leading to an instantaneous kick in the energy coordinate. The description enables conditions to be fulfilled for a linearized longitudinal focus - or a minimization of the energy spread. (It is, however, not limited to these two cases.) The analytic treatment is completed by a discussion of the longitudinal RMS emittance, which is a measure for the nonlinearities present in the phase space distribution of the bunch. Space charge forces are not treated in the mathematical approach.

The outcome of the analytical calculations is used as set of seed parameters - cavity amplitudes and phases-for numerical simulations, which are used to validate the method. First, a second order corrected bunching scheme is compared for similar settings with both tools-whereby ASTRA is used without space charge repulsion. In a second step-including space charge forces-a third order correction is attempted, requiring additional conditions to be fulfilled, which is achieved by the operation in the so-called overcompensation mode, a special case of the stretcher mode. Finally, in the last part of Sec. III, instead of a compressed bunch, which resembles an upright line in phase space, the capability to produce a bunch with extremely low energy spread, i.e., a horizontal line, is demonstrated.

For the calculations in the course of this article, a machine like REGAE is assumed, although the concept can, in principle, be generalized and adapted to other machines, as briefly discussed in Sec. IV. Regae is a conventional accelerator designed to perform timeresolved electron diffraction experiments [12], where the time resolution is given by the length of the bunch on target. Another goal-executed within the LAOLA collaboration at DESY - is the demonstration and analysis of the injection of a REGAE bunch into a laser-driven plasma wakefield [13]. The machine is currently being upgraded for this purpose and connected to a $200 \mathrm{TW}$ high power laser system.

REGAE consists of a 1.5 cell rf gun cavity (the cathode plane is located at $z_{\mathrm{G}}=0 \mathrm{~m}$ ) and a four cell buncher cavity $\left(z_{\mathrm{B}}=1.39 \mathrm{~m}\right)$, both operated at $3 \mathrm{GHz}$. The electron bunches are produced by ultraviolet light pulses of a frequency tripled titanium-sapphire laser which have an FWHM length of about 500 fs, i.e., around 200 fs RMS. The machine is designed to produce electron bunches of low charge $(Q \approx 100 \mathrm{fC})$, low transverse beam emittance $\left(\epsilon_{\mathrm{tr}}<100 \mathrm{~nm} \mathrm{rad}\right)$, and ultra-short length $\left(\sigma_{\mathrm{t}}<10 \mathrm{fs}\right)$ at the position of the target, $z_{\mathrm{F}}=5.50 \mathrm{~m}$ [2]. The geometry, which is depicted in Fig. 2, is used for all calculations in Sec. III, while the pulse length of the laser (and consequently the initial electron bunch length) is set to $\tau_{\text {RMS }}=1$ ps. The charge in the numerical simulations in Secs. III B and III D is only $50 \mathrm{fC}$ in contrast to the values quoted above.

The aim of this article is to present and demonstrate the validity of our approach, which can be realized in various configurations. An extensive feasibility study with tolerance analysis for the shortest bunches possible is beyond the scope.

\section{ANALYTICAL FORMULATION}

\section{A. Phase space development within a free drift}

The compression mechanism discussed here is completely treated in the longitudinal phase space of the bunch. Each particle has coordinates $(\zeta, \gamma)$, where $\gamma$ is the relativistic Lorentz factor and $\zeta$ is the relative longitudinal position in the bunch. The particle distribution is assumed to lie on a line, i.e., there is no uncorrelated energy spread.

The aim of the analytical treatment is to formulate a framework which yields parameter settings for the cavities which linearize the phase space at the location of the 
longitudinal focus. The formalism is based on two basic assumptions: (1) Within a drift of the bunch (segments II, IV of Fig. 2), the energy coordinate $\gamma$ is unchanged; space charge effects are neglected. (2) Within a cavity (segments I, III) the $\zeta$-coordinate does not change. (Thin lens approximation.)

The relative shift of the electrons in a drift is given by the velocity difference within the bunch. Since the velocity spread is based on the energy spread imprinted in an accelerating structure, a general formulation has to include the nonlinear evolution of the relative shift originating from the nonlinear correlation $\gamma=1 / \sqrt{1-\beta^{2}}$, where $\beta$ is the velocity (in units of speed of light). For a drift from $z_{0}$ to a point $z$ this can be expressed by [3]:

$$
\begin{aligned}
\Delta \zeta(z):= & \Delta v(z)\left(t(z)-t\left(z_{0}\right)\right)=\frac{1}{\beta} \Delta \beta(\gamma)\left(z-z_{0}\right) \\
= & \frac{1}{\bar{\beta}}\left[\left.\frac{\mathrm{d} \beta}{\mathrm{d} \gamma}\right|_{\bar{\gamma}} \delta \gamma+\left.\frac{1}{2} \frac{\mathrm{d}^{2} \beta}{\mathrm{d} \gamma^{2}}\right|_{\bar{\gamma}}(\delta \gamma)^{2}\right. \\
& \left.+\left.\frac{1}{6} \frac{\mathrm{d}^{3} \beta}{\mathrm{d} \gamma^{3}}\right|_{\bar{\gamma}}(\delta \gamma)^{3}+\cdots\right]_{z_{0}}\left(z-z_{0}\right),
\end{aligned}
$$

where $\bar{\beta}$ and $\bar{\gamma}$ are the velocity/energy of the central particle of the bunch $(\zeta=0)$ respectively, which marks the center of the Taylor expansion. $\delta \gamma$ denotes the energy difference from $\bar{\gamma}$.

The coefficients of this Taylor polynomial are given by:

$$
\begin{aligned}
& \eta_{1}(\bar{\gamma}):=\left.\frac{1}{\bar{\beta}} \frac{\mathrm{d} \beta}{\mathrm{d} \gamma}\right|_{\bar{\gamma}}=\frac{1}{\bar{\gamma}^{3} \bar{\beta}^{2}} \\
& \eta_{2}(\bar{\gamma}):=\left.\frac{1}{2 \bar{\beta}} \frac{\mathrm{d}^{2} \beta}{\mathrm{d} \gamma^{2}}\right|_{\bar{\gamma}}=\frac{2-3 \bar{\gamma}^{2}}{2 \bar{\gamma}^{6} \bar{\beta}^{4}} \\
& \eta_{3}(\bar{\gamma}):=\left.\frac{1}{6 \bar{\beta}} \frac{\mathrm{d}^{3} \beta}{\mathrm{d} \gamma^{3}}\right|_{\bar{\gamma}}=\frac{2-5 \bar{\gamma}^{2}+4 \bar{\gamma}^{4}}{2 \bar{\gamma}^{9} \bar{\beta}^{6}} .
\end{aligned}
$$

The consequence of the kinetics is that for any energy distribution that does not yield a linear velocity spread, a curvature and higher order distortions are generated in the longitudinal phase space of the bunch, solely due to the drift. The magnitude of these effects is strongly suppressed for high energies.

\section{B. Cavity effects on the phase space}

In addition, within the gun (or any other accelerating structure), the bunch acquires a curvature based on the shape of the accelerating field gradient, since the finite bunch length covers an interval $\pm \delta \phi$ around the nominal phase, $\phi$. A general description of this effect can be given again by a Taylor expansion of the respective function describing the energy gain within the cavity in dependence on the phase. In the cases treated here, the function for the gun originates from a numerical phase scan using ASTRA, which is fitted by a polynomial. An analytic calculation is difficult, due to the slippage of the electrons with respect to the phase of the field at the start [14]. The buncher, in contrast, is easily described by a sine function. Both equations can be expanded into a Taylor series in $\delta \phi$ around a phase $\phi$-which directly yields the change in the phase space coordinate $\gamma$. Using the correlation $\delta \phi=-\zeta k$, where $k$ represents the wave vector of accelerating field, the energy gain (or loss) can be expressed by

$$
\Delta \gamma\left(\zeta_{\mathrm{C}}\right)=\tilde{A}_{0}+\tilde{A}_{1} \zeta_{\mathrm{C}}+\tilde{A}_{2} \zeta_{\mathrm{C}}^{2}+\tilde{A}_{3} \zeta_{\mathrm{C}}^{3}+\cdots,
$$

where $\zeta_{\mathrm{C}}$ denotes the phase space coordinate at the position $z=z_{\mathrm{C}}$ of the cavity, and the $\tilde{A}_{i}$ are the respective Taylor coefficients.

If the incoming distribution $\hat{\gamma}$ is described in $\zeta_{\mathrm{C}}$, the resulting energy distribution after a cavity thus can be expressed in a polynomial form as well:

$$
\begin{aligned}
\gamma\left(\zeta_{\mathrm{C}}\right) & =\hat{\gamma}\left(\zeta_{\mathrm{C}}\right)+\Delta \gamma\left(\zeta_{\mathrm{C}}\right) \\
& =\underbrace{A_{0}}_{\bar{\gamma}}+\underbrace{A_{1} \zeta_{\mathrm{C}}+A_{2} \zeta_{\mathrm{C}}^{2}+A_{3} \zeta_{\mathrm{C}}^{3}+\cdots}_{\delta \gamma},
\end{aligned}
$$

where the coefficients $A_{i}=\hat{A}_{i}+\tilde{A}_{i}$ are determined by the sums of the coefficients of $\hat{\gamma}\left(\zeta_{\mathrm{C}}\right)$ and the $\tilde{A}_{i}$.

The resulting expressions for the energy of the central particle, $\bar{\gamma}$, and the energy spread $\delta \gamma$ can be inserted into Eq. (1) to describe the dynamics in the following drift. Since $\delta \gamma$ is a polynomial in $\zeta_{\mathrm{C}}$, the shifted coordinates due to the drift, $\zeta(z)=\zeta_{\mathrm{C}}+\Delta \zeta$ can be expressed in this cavity coordinates $\zeta_{\mathrm{C}}$. Multiplying out $\left(\delta \gamma\left(\zeta_{\mathrm{C}}\right)\right)^{2}$ and $\left(\delta \gamma\left(\zeta_{\mathrm{C}}\right)\right)^{3}$, neglecting terms of fourth and higher orders, and rearranging leads to

$$
\zeta(z)=\chi_{1}(z) \zeta_{\mathrm{C}}+\chi_{2}(z) \zeta_{\mathrm{C}}^{2}+\chi_{3}(z) \zeta_{\mathrm{C}}^{3}
$$

with the coefficients $\chi_{i}$ defined by

$$
\begin{aligned}
& \chi_{1}(z)=1+\left(z-z_{0}\right)\left[\eta_{1} A_{1}\right] \\
& \chi_{2}(z)=\left(z-z_{0}\right)\left[\eta_{1} A_{2}+\eta_{2} A_{1}^{2}\right] \\
& \chi_{3}(z)=\left(z-z_{0}\right)\left[\eta_{1} A_{3}+2 \eta_{2} A_{1} A_{2}+\eta_{3} A_{1}^{3}\right] .
\end{aligned}
$$

Equations (5) and (6) thus describe the evolution of the longitudinal phase space coordinate $\zeta$ of a particle along a drift section behind a cavity, depending on the start coordinates $\zeta_{\mathrm{C}}$ and the known, initial energy $\gamma\left(\zeta_{\mathrm{C}}\right)$ at the exit of the preceding cavity, which is determined by Eq. (4).

The magnitude of $\gamma$ as phase space coordinate is unchanged along the drift, since the energy is constant. But, the functional description-respecting the polynomial structure-must reflect the change in the position $\zeta$ of each particle. In that sense, Eq. (4) is a special case of a general expression, to which it can be equated, due to the constant magnitude of $\gamma$ : 


$$
\begin{aligned}
\gamma\left(\zeta_{\mathrm{C}}\right) & =A_{0}+A_{1} \zeta_{\mathrm{C}}+A_{2} \zeta_{\mathrm{C}}^{2}+A_{3} \zeta_{\mathrm{C}}^{3} \\
& \equiv a_{0}+a_{1} \zeta+a_{2} \zeta^{2}+a_{3} \zeta^{3}=\gamma(\zeta) .
\end{aligned}
$$

The capital letters are used for the coefficients at the cavity, in contrast to small letters which describe the development of the coefficients within a drift. This nomenclature will be used throughout the following derivation. Using Eq. (5) in the right-hand side of Eq. (7) yields the general coefficients

$$
\begin{aligned}
& a_{0}=A_{0}=\bar{\gamma} \quad a_{1}=\frac{A_{1}}{\chi_{1}} \\
& a_{2}=\frac{A_{2}-a_{1} \chi_{2}}{\chi_{1}^{2}}=\cdots=\frac{A_{2} \chi_{1}-A_{1} \chi_{2}}{\chi_{1}^{3}} \\
& a_{3}=\frac{A_{3}-a_{1} \chi_{3}-2 a_{2} \chi_{1} \chi_{2}}{\chi_{1}^{3}} .
\end{aligned}
$$

These coefficients compensate for the changes in $\zeta$, such that $\gamma$ is kept constant within the particle coordinates $(\zeta, \gamma(\zeta))$.

Equations (5) and (7), with the respective coefficients $\chi_{i}(z)$ and $a_{i}(z)$, characterize the phase space coordinates of each particle at an arbitrary bunch position $z$ along the drift after a cavity which defines the starting polynomial of $\gamma$ in $\zeta$. Thus, the phase space at the entrance of a subsequent cavity can be calculated.

\section{Start to end description: Iterative application}

Equations (3)-(8) provide, in principle, all tools to describe the development of the longitudinal phase space, and thus of the bunch length for an accelerator like REGAE. To generalize this formalism for such a machine with two cavities, as described in Sec. I, the following notation will be used: The parameters $A_{i}$ and $a_{i}$ of the energy polynomials [Eqs. (7) and (8)] are replaced by either $G_{i} / g_{i}$ for the electron gun, or $B_{i} / b_{i}$ for the buncher cavity. Capital letters denote the initial parameter set at the exit position of a cavity, while small letters are used for the subsequent drift, as stated above. Please note that, in the case of the gun, the distribution at the exit is taken from a numerical simulation, such that $G_{i}$ is directly known and serves as the starting distribution for the formalism.

Thus, to describe the whole system, the distribution arising from the gun and the drift toward the buncher cavity is evaluated at the position $z_{\mathrm{B}}$ of the buncher, leading to the phase space coordinates $\left(\zeta_{\mathrm{B}}, \hat{\gamma}\left(\zeta_{\mathrm{B}}\right)\right)$, where

$$
\begin{aligned}
\hat{\gamma}\left(\zeta_{\mathrm{B}}\right)= & g_{0}\left(z_{\mathrm{B}}\right)+g_{1}\left(z_{\mathrm{B}}\right) \zeta_{\mathrm{B}} \\
& +g_{2}\left(z_{\mathrm{B}}\right) \zeta_{\mathrm{B}}^{2}+g_{3}\left(z_{\mathrm{B}}\right) \zeta_{\mathrm{B}}^{3} .
\end{aligned}
$$

Using Eq. (4), the energy polynomial after the action of the buncher cavity is thus

$$
\begin{aligned}
\gamma_{\mathrm{B}}\left(\zeta_{\mathrm{B}}\right)= & \left(g_{0}+\tilde{B}_{0}\right)+\left(g_{1}+\tilde{B}_{1}\right) \zeta_{\mathrm{B}} \\
& +\left(g_{2}+\tilde{B}_{2}\right) \zeta_{\mathrm{B}}^{2}+\left(g_{3}+\tilde{B}_{3}\right) \zeta_{\mathrm{B}}^{3} \\
= & B_{0}+B_{1} \zeta_{\mathrm{B}}+B_{2} \zeta_{\mathrm{B}}^{2}+B_{3} \zeta_{\mathrm{B}}^{3} .
\end{aligned}
$$

Finally, from Eq. (10), the shift in the following drift can be calculated, applying the same pattern as above:

$$
\begin{aligned}
\zeta\left(z \geq z_{\mathrm{B}}\right) & =\zeta_{\mathrm{B}}+\Delta \zeta\left(z \geq z_{\mathrm{B}}\right) \\
& =X_{1} \zeta_{\mathrm{B}}+X_{2} \zeta_{\mathrm{B}}^{2}+X_{3} \zeta_{\mathrm{B}}^{3}
\end{aligned}
$$

with the coefficients

$$
\begin{aligned}
& X_{1}:=1+\left(z-z_{\mathrm{B}}\right)\left[H_{1} B_{1}\right] \\
& X_{2}:=\left(z-z_{\mathrm{B}}\right)\left[H_{1} B_{2}+H_{2} B_{1}^{2}\right] \\
& X_{3}:=\left(z-z_{\mathrm{B}}\right)\left[H_{1} B_{3}+2 H_{2} B_{1} B_{2}+H_{3} B_{1}^{3}\right],
\end{aligned}
$$

where $H_{i}$ are the coefficients $\eta_{i}$, Eq. (2), evaluated for the overall mean energy $\bar{\gamma}_{\mathrm{B}}=B_{0}=g_{0}+\tilde{B}_{0}$; all effects up to the buncher are already included in $g_{i} . H$ and $X$ are the label for a capital $\eta$ and $\chi$ respectively, to reflect that the same structure lies beneath the coefficients.

In a last step, analogous to (8), the coefficients for the function $\gamma_{\mathrm{B}}(\zeta)$ in the second drift can be determined, such that a complete description of the longitudinal phase space in that section is obtained-and such for the whole system. For the bunch length determination this evaluation is not required, since this only relies on the change in $\zeta$ which is already described by Eq. (11). However, the result is required for the calculation of the emittance. It is given by

$$
\begin{aligned}
\gamma_{\mathrm{B}}(\zeta) & =b_{0}+b_{1} \zeta+b_{2} \zeta^{2}+b_{3} \zeta^{3} \\
b_{0} & =B_{0}=\bar{\gamma}_{\mathrm{B}} \quad b_{1}=\frac{B_{1}}{X_{1}} \\
b_{2} & =\frac{B_{2}-b_{1} X_{2}}{X_{1}^{2}}=\cdots=\frac{B_{2} X_{1}-B_{1} X_{2}}{X_{1}^{3}} \\
b_{3} & =\frac{B_{3}-b_{1} X_{3}-2 b_{2} X_{1} X_{2}}{X_{1}^{3}} .
\end{aligned}
$$

\section{Summary}

In the following, a summary of the general approach is given-with the bullets corresponding to the respective segments in Fig. 2 and the phase space coordinates $(\zeta(z), \gamma(\zeta ; z))$ :

(I) The phase space at the gun is characterized by the start distribution in $\zeta_{\mathrm{G}} \equiv \zeta\left(z_{\mathrm{G}}\right)$ and the corresponding $\gamma$-coordinate from a simulation, which can be expressed as a function of $\zeta_{\mathrm{G}}$. It is expanded into a Taylor polynomial around the nominal phase of the cavity field, where the central particle is located. The gun polynomial yields the coefficients $G_{i}$. 


$$
\left(\zeta_{\mathrm{G}}, \sum G_{i} \zeta_{\mathrm{G}}^{i}\right)
$$

(II) The start configuration from above is typically curved, and acquires an additional curvature in the subsequent drift due to the nonlinear correlation of energy and velocityincluded in the $\eta_{i}$ factors of Eq. (1). The phase space coordinate $\zeta(z)$ for each particle, depending on the bunch position $z$, is described by Eq. (5)-a polynomial in the start coordinate $\zeta_{\mathrm{G}}$ and the $\chi_{i}(z)$ coefficients [Eqs. (6)], which include the nonlinear correlations as well as the $z$ dependence. To describe the phase space $\gamma(\zeta ; z)$ as a function of $z$, the change in this function is calculatedmaking use of the fact that the magnitude of $\gamma$ for each particle is unchanged, leading to Eq. (7) with the $g_{i}(z)$ coefficients analogous to Eq. (8).

$$
\left(\sum \chi_{i}(z) \zeta_{\mathrm{G}}^{i}, \sum g_{i}(z) \zeta(z)^{i}\right)
$$

(III) Evaluating the phase space at the buncher position $z=z_{\mathrm{B}}$ enables the application of this cavity's field by a simple summation of the evolved phase space function $\hat{\gamma}\left(\zeta_{\mathrm{B}}\right)$ and the effect of the cavity field $\Delta \gamma\left(\zeta_{\mathrm{B}}\right)$ [Eq. (4)], which amounts to the sums of the evolved coefficients $g_{i}\left(z_{\mathrm{B}}\right)$ and buncher coefficients $\tilde{B}_{i}$ [Eq. (10)], denoted by $B_{i}$. The transported $\zeta\left(z_{\mathrm{B}}\right)=\zeta_{\mathrm{B}}$ coordinates are unchanged in this region, since the cavity is treated as a thin lens: The action of the buncher amounts to an instantaneous kick in this approximation.

$$
\left(\sum \chi_{i}\left(z_{\mathrm{B}}\right) \zeta_{\mathrm{G}}^{i}, \sum B_{i}\left(z_{\mathrm{B}}\right) \zeta_{\mathrm{B}}^{i}\right)
$$

(IV) Knowing the phase space after the action of the second cavity as described above, the same formalism as in (II) is applied in the second drift. The $\zeta(z)$ coordinate is described by a polynomial in the $\zeta_{\mathrm{B}}$ coordinates at the buncher. The corresponding coefficients $X_{i}$ are simply Eqs. (6) evaluated for the evolved phase space at $z_{\mathrm{B}}$. In a similar manner, the phase space function $\gamma_{\mathrm{B}}(z)$ can also be determined, keeping the magnitude of $\gamma_{\mathrm{B}}$ for each particle constant, which results in Eq. (13) with the coefficients $b_{i}(z)$.

$$
\left(\sum X_{i}(z) \zeta_{\mathrm{B}}^{i}, \sum b_{i}(z) \zeta(z)^{i}\right) .
$$

(V) Since with (IV) the phase space can be determined in the corresponding second drift, the $\zeta$-coordinate of each particle at the focus position $z_{\mathrm{F}}$-which marks the end of region (IV) - can be determined by evaluating Eq. (11) with the coefficients $X_{i}$ at $z_{\mathrm{F}}$. The energy function typically can no longer by calculated at this point, since the description of the phase space as a function breaks down due to ambiguities. However, each particle's
$\gamma=\gamma_{\mathrm{B}}$ is known, since-once again-the magnitude of $\gamma$ is unchanged in a drift. Hence, the phase space still can be depicted.

$$
\left(\sum X_{i}\left(z_{\mathrm{F}}\right) \zeta_{\mathrm{B}}^{i}, \sum B_{i}\left(z_{\mathrm{B}}\right) \zeta_{\mathrm{B}}^{i}\right)
$$

With the formulation in the last step, a closed description from gun to focus is achieved. It maps the start coordinates $\zeta_{\mathrm{G}}$ onto the respective coordinate at the focus position, $\zeta\left(z_{\mathrm{F}}\right)=\zeta_{\mathrm{F}}$ (or at any other position in between). The optimal focus-a straight, perpendicular line in phase space-is achieved, when $\zeta_{\mathrm{F}}=0$ for all $\zeta_{\mathrm{G}}$. This happens if the $X_{i}$ coefficients vanish simultaneously. Since they only depend on the parameters $G_{i}$ and $B_{i}$, which are functions of the cavity amplitudes $E_{\mathrm{G}}, E_{\mathrm{B}}$ and phases $\phi_{\mathrm{G}}, \phi_{\mathrm{B}}$, there are four (and thus sufficient) free parameters to solve three equations. The solution is independent of the start distribution, as far as the phase space can be approximated as a line.

Finally, please note, that a real higher harmonic cavity can be implemented into the analytic calculations as well.

\section{E. Longitudinal emittance}

A measure for the nonlinearities present in the longitudinal phase space distribution is the longitudinal RMS emittance. This value is not the phase space volume (and thus a constant of motion), but includes phase space correlations of second and higher orders. It is given by

$$
\epsilon_{1, \mathrm{RMS}}=\sqrt{\left\langle\zeta^{2}\right\rangle\left\langle E_{\mathrm{kin}}^{2}\right\rangle-\left\langle\zeta E_{\mathrm{kin}}\right\rangle^{2}}
$$

where $\left\langle\cdot{ }^{2}\right\rangle$ defines the second central moment or variance of the (normalized) density distribution, $\rho$, while the second term is the co-variance of $\rho$, which removes the linear correlations from the RMS emittance calculation [14]:

$$
\begin{aligned}
\left\langle f(x)^{2}\right\rangle= & \int \rho(x) f(x)^{2} \mathrm{~d} x-\left(\int \rho(x) f(x) \mathrm{d} x\right)^{2} \\
\langle f(x) g(x)\rangle= & \int \rho(x) f(x) g(x) \mathrm{d} x \\
& -\int \rho(x) f(x) \mathrm{d} x \int \rho(x) g(x) \mathrm{d} x .
\end{aligned}
$$

The emittance can be determined for a discrete distribution, like an electron bunch in a simulation, by evaluating the corresponding sums over all particle coordinates present in the simulation. This approach is also possible and partly used in the course of this article, since the function $\gamma(\zeta)=E_{\text {kin }} / E_{\text {rest }}+1$ is evaluated and visualized for a finite particle set; $E_{\text {kin }}$ and $E_{\text {rest }}$ denote the kinetic energy and rest energy of an electron.

However, more insight can be gained by applying the analytic approach from above. From Eq. (14) one can deduce that the longitudinal RMS emittance is proportional 
to the product of the bunch length and the nonlinear energy spread [14]:

$$
\epsilon_{1, \mathrm{RMS}} \propto \sqrt{\left\langle\delta \gamma_{\mathrm{nl}}^{2}\right\rangle\left\langle\zeta^{2}\right\rangle}=\delta \gamma_{\mathrm{nl}, \mathrm{RMS}} \sigma_{\zeta}
$$

where the nonlinear energy spread $\delta \gamma_{\mathrm{nl}}$ is given by:

$$
\delta \gamma_{\mathrm{nl}}=\delta \gamma-\frac{\langle\zeta \delta \gamma\rangle}{\left\langle\zeta^{2}\right\rangle} \zeta
$$

From this, the emittance can be calculated directly, if the density distribution $\rho(\zeta)$ is known. For example, assuming a uniform density distribution,

$$
\rho(\zeta)=\left\{\begin{array}{cl}
\frac{1}{2 \zeta_{\mathrm{m}}}, & \text { for }|\zeta| \leq \zeta_{\mathrm{m}} \\
0, & \text { else },
\end{array}\right.
$$

the nonlinear energy spread according to Eq. (18) and the integral calculus above amounts to

$$
\delta \gamma_{\mathrm{nl}}=a_{2} \zeta^{2}+a_{3} \zeta^{3}-\frac{3}{5} a_{3} \zeta_{\mathrm{m}}^{2} \zeta
$$

i.e., the nonlinear terms of the energy polynomial (7), minus a correction that removes the linear contribution of the third order.

It is straightforward to calculate $\sigma_{\zeta}=\sqrt{1 / 3} \zeta_{\mathrm{m}}$, and finally the RMS of the nonlinear energy spread results in

$$
\begin{aligned}
\delta \gamma_{\mathrm{nl}, \mathrm{RMS}}= & {\left[\frac{1}{2 \zeta_{\mathrm{m}}} \int_{-\zeta_{\mathrm{m}}}^{\zeta_{\mathrm{m}}}\left(a_{2} \zeta^{2}+a_{3} \zeta^{3}-\frac{3}{5} a_{3} \zeta_{\mathrm{m}}^{2} \zeta\right)^{2} \mathrm{~d} \zeta\right.} \\
& \left.-\left(\frac{1}{3} a_{2} \zeta_{\mathrm{m}}^{2}\right)^{2}\right]^{\frac{1}{2}} \\
= & \sqrt{\frac{4}{45} a_{2}^{2} \zeta_{\mathrm{m}}^{4}+\frac{4}{175} a_{3}^{2} \zeta_{\mathrm{m}}^{6}} \approx \frac{2}{\sqrt{5}}\left|a_{2}\right| \sigma_{\zeta}^{2} .
\end{aligned}
$$

The second term in the square brackets originates from the mean of $\delta \gamma$-which differs by this value from $\bar{\gamma}$, describing the energy of the central particle of the distribution in $\zeta$. The proportionality of $\delta \gamma_{\mathrm{nl}, \mathrm{RMS}} \propto\left|a_{2}\right| \sigma_{\zeta}^{2}$ in the leading order is valid for any symmetric distribution, with the constant of proportionality depending on the kind of distribution. Hence, for such conditions, the longitudinal RMS emittance at a certain point $z$ can be described as

$$
\begin{aligned}
\epsilon_{1, \mathrm{RMS}}(z) & =D(z)\left|a_{2}(z)\right| \sigma_{\zeta}(z)^{3} \\
& \approx D(z)\left|a_{2}(z) \chi_{1}(z)^{3}\right| \zeta_{\mathrm{C}, \mathrm{RMS}}^{3} \\
& =D(z)\left|A_{2} \chi_{1}(z)-A_{1} \chi_{2}(z)\right| \zeta_{\mathrm{C}, \mathrm{RMS}}^{3}
\end{aligned}
$$

Here, $D(z)$ denotes the constant of proportionality and $\zeta_{\mathrm{C}, \mathrm{RMS}}:=\sigma_{\zeta}\left(z_{\mathrm{C}}\right)$ is the RMS of the distribution at the gun.
The equality $\sigma_{\zeta}(z)=\chi_{1}(z) \zeta_{\mathrm{C}, \mathrm{RMS}}$ is a strong assumption: It demands that the particle at the RMS position of the initial particle distribution stays at the RMS position of the bunch along the drift. This is only fulfilled for linear changes (which are exactly described by $\chi_{1}(z)$ ); deviations, however, are order $\zeta^{4}$ and thus neglected per definition. This also implies that the kind of distribution does not change in the drift in the leading order, keeping $D(z) \equiv D$ also constant in $z$. For a more accurate description, one could absorb changes in the distribution into $D(z)$, as long as the symmetry is not spoiled; this also implies a certain independence of the functional description of the emittance from the distribution - which is based on the fact that the functional description of the drift behavior itself does not depend on the distribution. The validity of Eq. (21) breaks down close to the focus, since around that point the density distribution $\rho$ is no longer symmetric.

The main point of the emittance discussion lies in the term $\left|A_{2} \chi_{1}(z)-A_{1} \chi_{2}(z)\right|$. Since this is proportional to $\left|a_{2}\right|$, $\epsilon_{1, \text { RMS }}(z)$ describes the behavior of the second order. It thus has to vanish-if evaluated in leading order-at the focal spot $z_{\mathrm{F}}$, if the second order should be corrected. (Since higher orders are still present, and the symmetry breaks down, in general it will have a local minimum, where the magnitude of $\epsilon_{1, \mathrm{RMS}}\left(z_{\mathrm{F}}\right)$ is determined by the higher order nonlinearities.)

\section{APPLICATION}

\section{A. Optimized bunching: Second order correlations}

To illustrate the functionality of the approach described above, an optimized case (i) is chosen from a numerical solution of Eqs. (12), where $X_{1}$ and $X_{2}$ are set to zero at $z=z_{\mathrm{f}}=5.50 \mathrm{~m} . X_{1 / 2}=0$ ensures that during the drift all particles are shifted such that they reach $\zeta=0$ at the same time at $z_{\mathrm{f}}$. Hence, the beam is focused longitudinally up to the second order. The parameter set determined is $E_{\mathrm{G}}(\mathrm{i})=100.0 \mathrm{MV} / \mathrm{m}, \phi_{\mathrm{G}}(\mathrm{i})=34.1 \mathrm{deg}$ and $E_{\mathrm{B}}(\mathrm{i})=$ 21.2 MV/m, $\phi_{\mathrm{B}}(\mathrm{i})=-109.3 \mathrm{deg}$, resulting in a beam with an energy of about $E_{\text {kin }}(\mathrm{i})=3.2 \mathrm{MeV}$. $\left(\phi_{\mathrm{G}}=\right.$ $\phi_{\mathrm{B}}=0.0$ corresponds to the maximum accelerating gradient of the respective cavity.)

Case (i) is now compared with two scenarios. On the one hand, case (ii), the gun amplitude is kept at $E_{\mathrm{G}}(\mathrm{ii})=$ $100.0 \mathrm{MV} / \mathrm{m}$, while for case (iii) the gun amplitude is set to $E_{\mathrm{G}}($ iii $)=70.0 \mathrm{MV} / \mathrm{m}$. For both cases, the gun phase is set to the maximum accelerating phase $\left(\phi_{\mathrm{G}}(\mathrm{ii}, \mathrm{iii})=0.0 \mathrm{deg}\right)$ and the buncher cavity is adjusted to $\phi_{\mathrm{B}}(\mathrm{ii}, \mathrm{iii})=-90.0 \mathrm{deg}$. The amplitudes of the buncher cavity are tuned to $E_{\mathrm{B}}(\mathrm{ii})=17.6 \mathrm{MV} / \mathrm{m}$ and $E_{\mathrm{B}}(\mathrm{iii})=$ $6.6 \mathrm{MV} / \mathrm{m}$ respectively, resulting in a bunched beam at $z_{\mathrm{F}}$. The kinetic energy of these beams is $E_{\text {kin }}$ (ii) $=4.6 \mathrm{MeV}$ and $E_{\text {kin }}($ iii $)=3.2 \mathrm{MeV}$. The phase settings are illustrated in Figs. 3 and 4. Figure 5 shows a comparison of the calculation from the respective longitudinal phase 


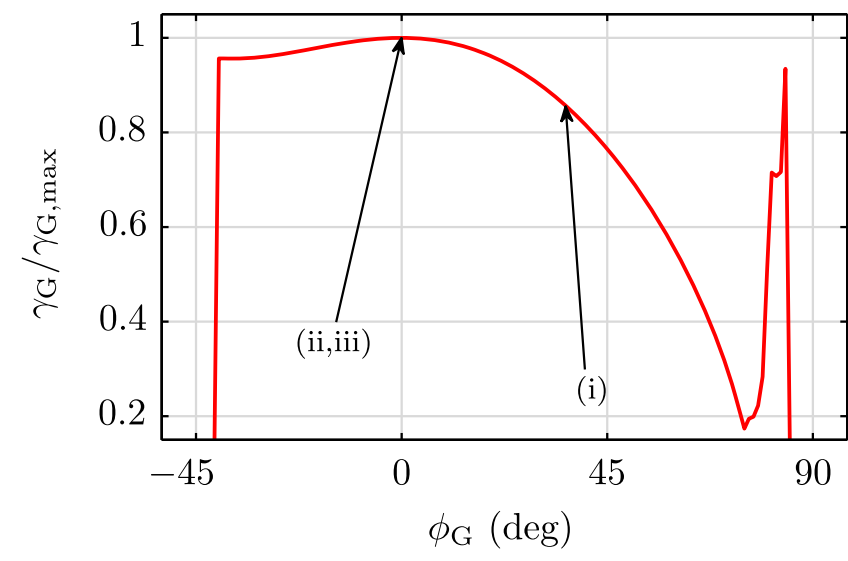

FIG. 3. Phase scan of the gun cavity. For case (i), an expanding phase is chosen, while for the comparisons (ii,iii) the maximum accelerating phase, $\phi_{\mathrm{G}}=0$, is applied.

spaces at the focal spot. For cases (ii) and (iii), a clear signature of the quadratic term is present, which limits the minimal bunch length. According to the calculation, the RMS bunch lengths are $\sigma_{\zeta}(\mathrm{ii})=6.6 \mu \mathrm{m} \widehat{=} 22 \mathrm{fs}$ and $\sigma_{\zeta}(\mathrm{iii})=5.9 \mu \mathrm{m} \widehat{=} 20 \mathrm{fs}$. In contrast, for case (i) the quadratic contribution to the curvature is eliminated, leading to a much shorter bunch of $\sigma_{\zeta}(\mathrm{i})=230 \mathrm{~nm} \widehat{=} 770$ as, limited by the third order.

The longitudinal density distribution at the start, which mimics the temporal distribution of the laser at the cathode surface, is set to an inverted parabola for all cases treated in this article. This distribution is suitable to reduce space charge effects [3], which is relevant for case (iv) in Sec. III B and the energy spread compensation scheme discussed in Sec. III D. The RMS length at the cathode is

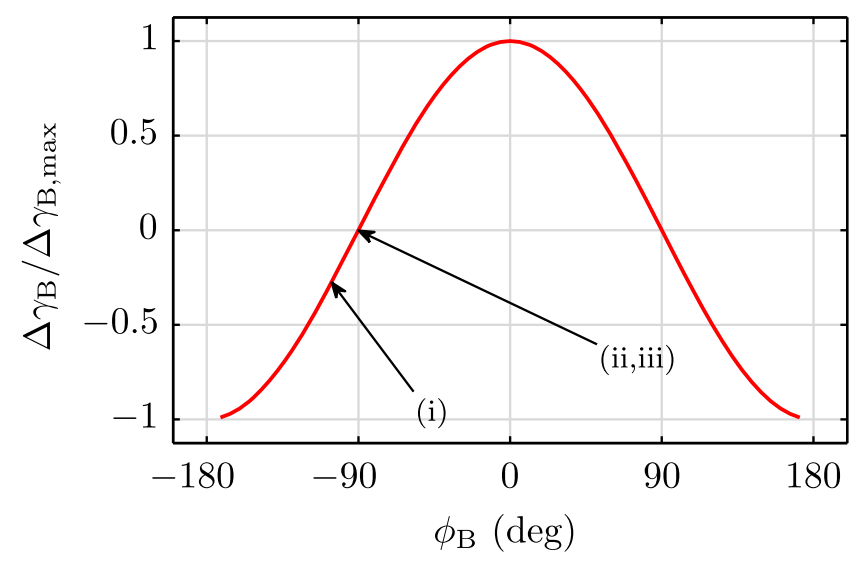

FIG. 4. Calculated energy gain of the buncher cavity in dependency of the phase. For case (i) a decelerating phase is set, determined by the analytic model for a curvature corrected focus at $z=5.50 \mathrm{~m}$. Cases (ii,iii) are set to the zero crossing and the amplitude is tuned for a bunch length minimum at the same focus position.

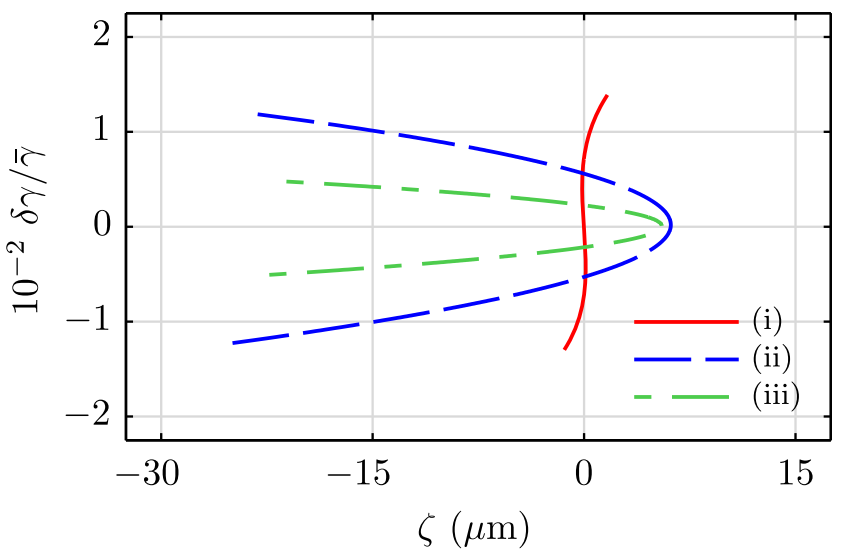

FIG. 5. Comparison of the calculated phase space distributions in the focus for cases (i,ii,iii). Cases (ii,iii) are clearly limited by the second order in contrast to scenario (i), which is much shorter. It is almost a straight, upright line with a signature of the third order.

kept at $\sigma_{t}=1 \mathrm{ps}$ for the AstRA simulations. For the analytic calculations, this value has to be multiplied by the bunch compression factor of the gun [14] to achieve the same start conditions for both models, since this factor corrects the initial bunch length for changes within the gun. Comparing the value at the cathode to the bunch length in the focus, one obtains a compression factor of the second order corrected scheme which is larger than 1000.

To further demonstrate the validity of the approach, it is analyzed by AsTRA studies (without space charge). The parameters need to be slightly adjusted in ASTRA to account for deviations arising from the thin lens approximation of the buncher in the numerical approach, as well as for gun dynamics and transverse effects. The mathematical model in that sense yields seed parameters for the particle tracker. Figure 6 shows the evolution of case (i) in ASTRA and the mathematical model, as well as the calculated bunch length along the beamline for case (iii). The mathematical approach yields a very close result compared to the particle tracker.

As can be seen, for (i) the electron bunch is first stretched until it reaches the buncher cavity, which now acts as a pseudoharmonic structure: Due to the significant lengthening, the electron bunch covers a phase interval $\delta \phi_{\mathrm{B}}$ that differs from the phase interval $\delta \phi_{\mathrm{G}}$ at the gun. Hence, for $\delta \phi_{\mathrm{B}}>\delta \phi_{\mathrm{G}}$-i.e., a decompression within the first drift-the second cavity effectively has a shorter wavelength from the point of view of the bunch compared to the gun, despite the fact that both cavities are operated at the same frequency of $3 \mathrm{GHz}$. As a consequence, the electrons are decelerated in the buncher cavity $\left(E_{\mathrm{kin}}(\mathrm{i})=4.0 \mathrm{MeV}\right.$ down to $E_{\text {kin }}(\mathrm{i})=3.1 \mathrm{MeV}$ ) and longitudinally focused-including the second order, similar to a third harmonic system $[3,8]$. For case (iii), in contrast, there is no bunch length evolution visible up to the buncher, since the particles originate from the on-crest phase of the gun where the slope is zero; the minimal bunch length is limited by the second order. 

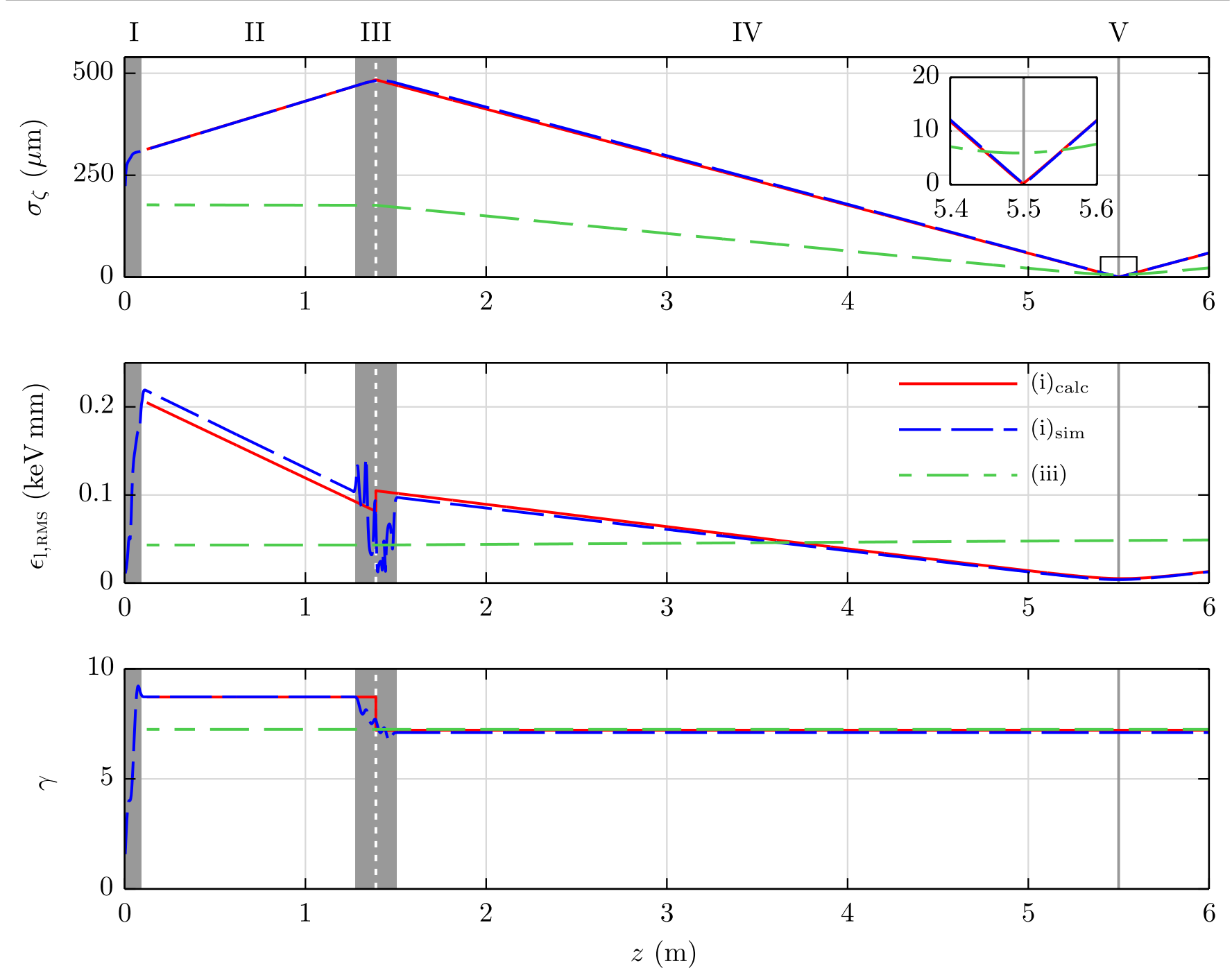

FIG. 6. Evolution of the bunch length $\sigma_{\zeta}(z)$, the longitudinal emittance $\epsilon_{1, \mathrm{RMS}}(z)$ and the energy $\gamma(z)$ along the beam line for the case (i) - analytical calculation and simulation — and case (iii), calculation only. Both versions of (i) are in very good agreement. The bunch length in (i) is first increasing, since the gun is set for an expanding phase, in contrast to (iii), where it does not change. The position of the minimum coincides for all cases, but the bunch is shorter for case (i) in the minimum. The emittance has a local minimum at the same position, indicating the vanishing of the second order of (i), while in (iii) there is almost no change. The bunch energy for case (iii) stays constant as well, while in (i) a deceleration takes place in the buncher.

As discussed, a good measure for the minimization of higher orders is the longitudinal RMS emittance. Elimination of the second order curvature at a certain point should lead to a minimum of the RMS emittance at that spot. Thus, matching this minimum to the minimum bunch length should yield a focused, second order corrected phase space distribution. As can be seen from Fig. 6, this condition holds for case (i), while the longitudinal RMS emittance for (iii) is slightly increasing behind the buncher cavity. Note that the longitudinal emittance is in general not constant in a drift due to the dynamics described by Eq. (2). For case (i) an overcompensation of the second order nonlinearity is hence required in the buncher cavity (compare with [3]).
A direct comparison of case (i) calculated with the analytical model and the according ASTRA simulation with similar parameters is presented in Fig. 7, showing four snapshots of the longitudinal phase space. The analytic method models the resulting bunch structure very well. Both phase space distributions are taken at four positions around the shortest bunch length. The RMS focus in both cases is actually at an offset $\Delta z=-2 \mathrm{~mm}$ in front of the ideal spot $z_{\mathrm{F}}$ : Due to the dominant third order presentwhich determines the S-shape-the RMS bunch length is shorter at this position, since the third order effect is partly compensated by a small first order, such that the slope is not yet completely perpendicular. The minimal RMS extent of the beam for the simulation is even shorter 

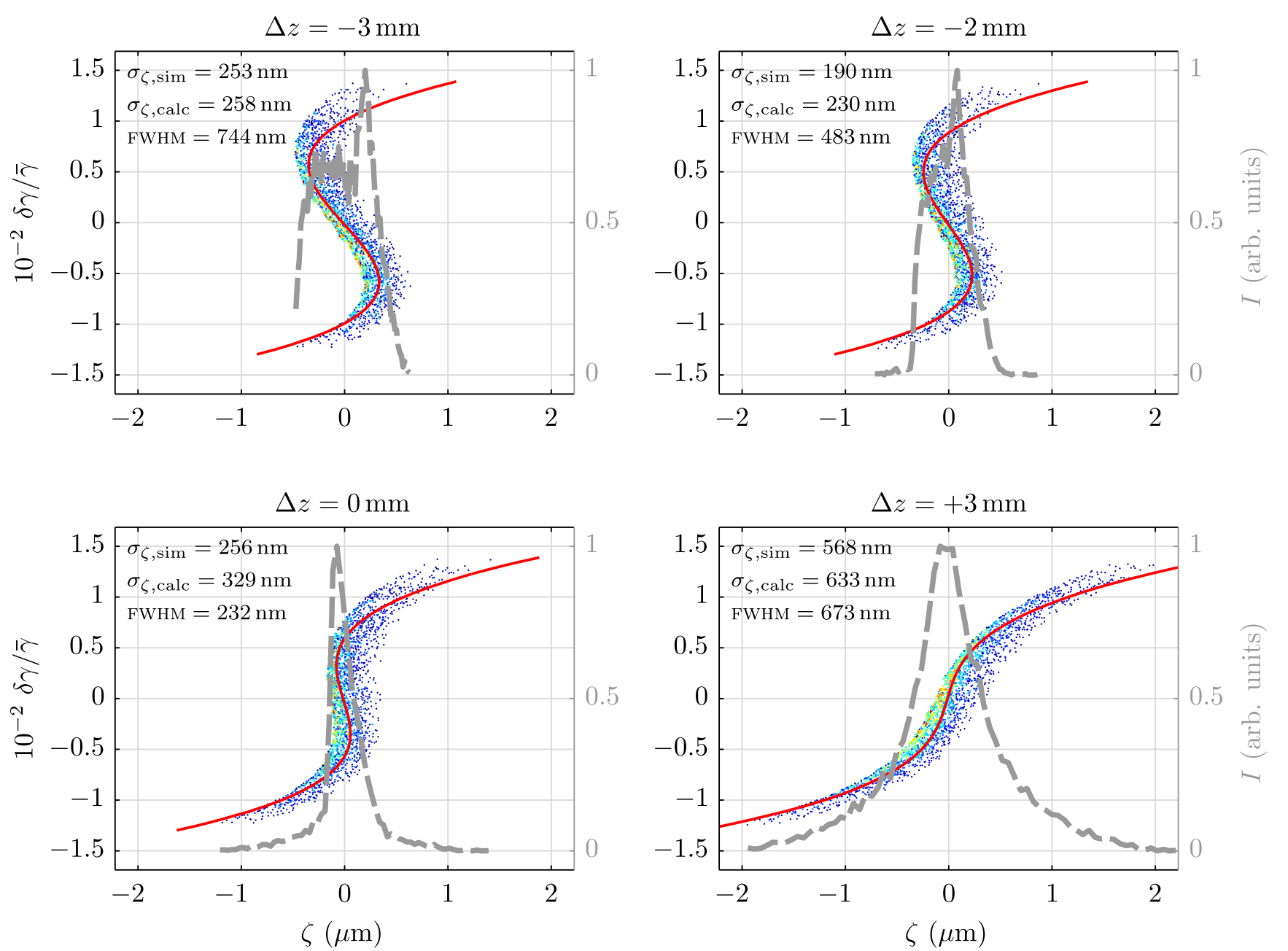

FIG. 7. Comparison of the longitudinal phase space of case (i) at four different positions around the shortest bunch length. For both versions, an inverted parabolic density distribution with an emission time of $\tau_{\mathrm{RMS}}=1 \mathrm{ps}$ is launched. The analytic calculation, depicted by the red line, resembles the tracked distribution (without space charge) to a very good degree-despite the thin lens approximation of the buncher description. The gray dashed line depicts the current profile of the distribution, determined by the local particle density of the ASTRA simulation results. At $\Delta z=0 \mathrm{~mm}$, the slope is (almost) infinite, which resembles the focus definition of the analytical model: all particles have the same longitudinal position at the same time (up to the second order). At this position, the FWHM width-evaluated from the current profile-is minimal. The minimal RMS bunch length is reached at $(\Delta z=-2 \mathrm{~mm})$, since the third order present is partly compensated by a negative slope.

with $\sigma_{\zeta}(\mathrm{i})_{\text {sim }}=190 \mathrm{~nm} \widehat{=} 630$ as compared to $\sigma_{\zeta}(\mathrm{i})_{\text {calc }}=$ $230 \mathrm{~nm}$. In contrast, at $\Delta z=0 \mathrm{~mm}$ - the location where the slope is nearly infinite, corresponding to the definition of the longitudinal focus of the analytic treatment-the
FWHM width of the beam is minimal. It has a size of $\mathrm{FWHM}=232 \mathrm{~nm}$. Due to the slight difference in parameters, the mean energy of the simulated bunch is about $80 \mathrm{keV}$ smaller than predicted by the analytical model. A

TABLE I. Overview of the parameter settings for the different bunching configurations discussed.

\begin{tabular}{lcccccrccc}
\hline \hline Case & Method & Space charge & $E_{\mathrm{G}}(\mathrm{MV} / \mathrm{m})$ & $\phi_{\mathrm{G}}(\mathrm{deg})$ & $E_{\mathrm{B}}(\mathrm{MV} / \mathrm{m})$ & $\phi_{\mathrm{B}}(\mathrm{deg})$ & $E_{\mathrm{kin}}(\mathrm{MeV})$ & $\sigma_{\zeta}\left(z_{\mathrm{F}}\right)(\mu \mathrm{m})$ & $\sigma_{t}\left(z_{\mathrm{F}}\right)(\mathrm{fs})$ \\
\hline (i) & analytic & & 100.00 & 34.13 & 21.21 & -109.29 & 3.17 & 0.23 \\
(i) & simulation & no & 100.00 & 34.13 & 21.66 & -111.99 & 3.12 & 0.77 \\
(ii) & analytic & & 100.00 & 0.00 & 17.60 & -90.00 & 4.60 & 6.58 & 21.95 \\
(iii) & analytic & & 70.00 & 0.00 & 6.60 & -90.00 & 3.19 & 5.89 & 19.66 \\
(iv) & analytic & & 100.00 & 42.75 & 20.94 & -94.45 & 3.35 & 0.01 & 0.04 \\
(iv) & simulation & no & 100.00 & 38.00 & 21.77 & -104.15 & 3.25 & 0.11 \\
(iv) & simulation & 50 fC & 100.00 & 42.00 & 21.63 & -97.42 & 3.32 & 0.24 & 0.38 \\
\hline \hline
\end{tabular}


comparison of the parameters used in all the different cases, as well as the results, are given in Table I.

\section{B. Optimized bunching: Third order correlations and space charge}

So far, only the second order has been corrected. Since only the buncher settings have been used for this, only two of the four free parameters (phases and amplitudes of the two cavities) have been used. A scan through the gun phase, while still optimizing buncher phase and amplitude for a second order corrected focus at $z_{\mathrm{F}}=5.50 \mathrm{~m}$, yields a set of parameter combinations for gun and buncher. Evaluating the third order coefficient, $X_{3}$ of Eq. (12), for these settings shows a change of sign at around $\phi_{\mathrm{G}}=43$ deg for the case of $E_{\mathrm{G}}=100.0 \mathrm{MV} / \mathrm{m}$; see Fig. 8. Hence, there are settings - case (iv) - for which the third order vanishes as well.

Case (iv) is determined by $E_{\mathrm{G}}=100.0 \mathrm{MV} / \mathrm{m}, \phi_{\mathrm{G}}=$ $42.8 \mathrm{MV} / \mathrm{m}$ and $E_{\mathrm{B}}=20.9 \mathrm{MV} / \mathrm{m}, \phi_{\mathrm{G}}=-94.5 \mathrm{deg}$. A comparison of the longitudinal phase space of case (i) with the latter at the focus $z_{\mathrm{F}}$ reflects this behavior, as can be seen from Fig. 9. The change of sign of the third order term becomes evident in the transition from an $\mathrm{S}$-shape in case (i) to a Z-shape in case (iv). Since the longitudinal phase space is described by a line and only correlations up to third order are taken into account in our analytical model, the theoretical bunch length approaches zero for a complete correction. In the simulation, the bunch length becomes completely dominated by the uncorrelated energy spread and space charge repulsion with the third order correction in place. Testing and optimizing these parameters with ASTRA (without space charge) leads to a bunch length of about $115 \mathrm{~nm}$ which is an improvement of about $50 \%$ compared to case (i).

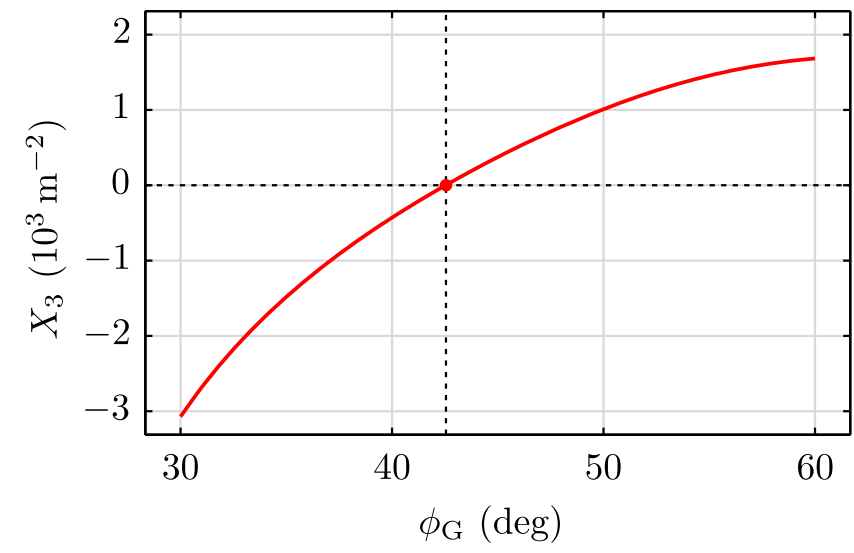

FIG. 8. The coefficient $X_{3}$, describing the third order shift in $\zeta$ at $z=z_{\mathrm{F}}$, solved for parameter sets determined by $X_{1}\left(z_{\mathrm{F}}\right)=$ $X_{2}\left(z_{\mathrm{F}}\right)=0$ for a gun amplitude $E_{\mathrm{G}}=100 \mathrm{MV} / \mathrm{m}$ and gun phase $\phi_{\mathrm{G}}$ varying from about $30 \mathrm{deg}$ to $60 \mathrm{deg} . X_{3}$ is negative for smaller values and crosses zero at about $43 \mathrm{deg}$, where a change of sign happens. The third order contribution should hence vanish for $\phi_{G} \approx 43 \mathrm{deg}$ as well.

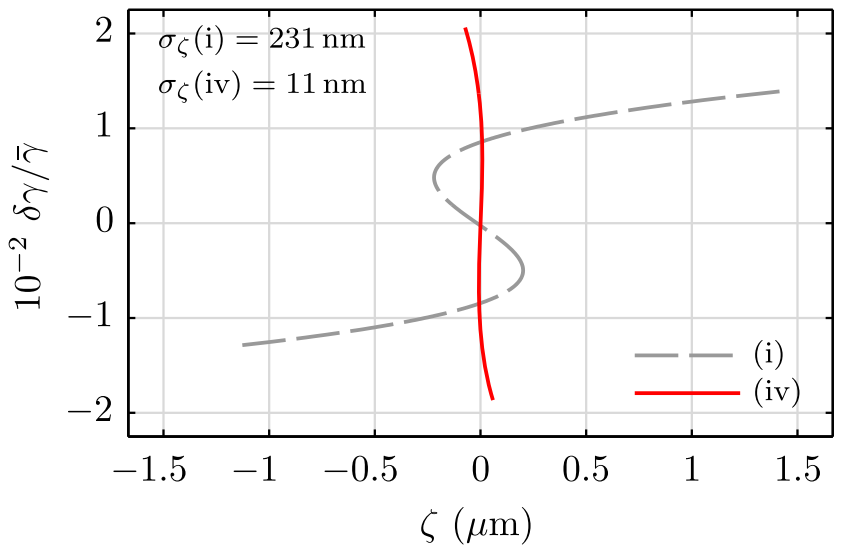

FIG. 9. Comparison of case (i) and (iv) at the focus position $z_{\mathrm{F}}=5.50 \mathrm{~m}$. The second order contribution vanishes in both cases, however, in case (i) a strong, negative third order is visibleexpressed by the S-shaped phase space figure. In case (iv), the magnitude of the third order is much less; in addition, the S-shape changed to $\mathrm{Z}$, meaning that $X_{3}$ is slightly positive for the parameter set chosen.

Adding space charge to the simulations again requires some parameter tweaking. To keep space charge effects low, a charge of $50 \mathrm{fC}$ has been assumed, which is a typical value for diffraction experiments at REGAE [12]. The shortest bunch length with these parameters achieved here is about $240 \mathrm{~nm}$, just below $1 \mathrm{fs}$. Further parameter scans could even improve this value. The phase space plot in focus, Fig. 10, clearly shows signs of space charge repulsion. The compression factor even with space charge is still larger than 1000 .

\section{Overcompensation mode}

It is interesting to note that, in order to remove the third order, the second order $\left(g_{2}\right)$ has to change sign before the bunch enters the buncher cavity:

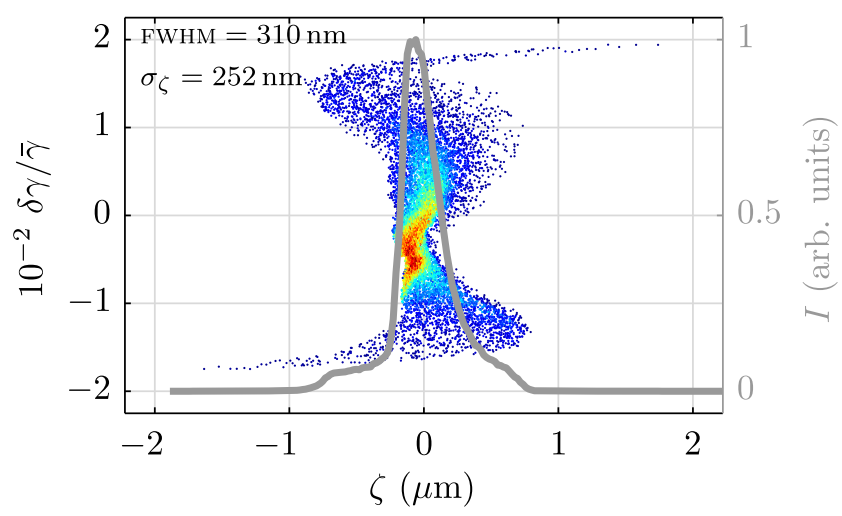

FIG. 10. Longitudinal phase space of an ASTRA simulation close to the focus. The bunch length in the focus with an eliminated third order and a charge of $Q=50 \mathrm{fC}$ is about $\sigma_{\zeta}=240 \mathrm{~nm}$, corresponding to 800 as. The prominent wings result from space charge repulsion happening mostly in the gun. 
$X_{3}$ can be expressed as

$$
\begin{aligned}
X_{3} & =0=H_{1} B_{3}+2 H_{2} B_{1} B_{2}+H_{3} B_{1}^{3} \\
& =\cdots=H_{1} B_{3}+\underbrace{\left(H_{3}-2 \frac{H_{2}^{2}}{H_{1}}\right)}_{<0} B_{1}^{3} .
\end{aligned}
$$

In order to achieve a bunching configuration, $B_{1}=g_{1}+$ $\tilde{B}_{1}$ needs to be negative, since this describes the slope in the phase space. This implies two things: First of all, $\tilde{B}_{1}<0$, since $g_{1}$ is positive to achieve the expansion in Sec. II. Second, the second term in Eq. (22) is positive, meaning that $H_{1} B_{3}$ must be negative, resulting in $B_{3}<0$, since $H_{1}=\gamma^{-3} \beta^{-2}$ is always positive. Since $\tilde{B}_{3}$ is proportional to the second derivative of $\tilde{B}_{1}$, which is given by a sine function, $\tilde{B}_{3}$ has always the opposite sign of $\tilde{B}_{1}$. Thus, $\tilde{B}_{3}$ needs to be positive and, from $B_{3}=g_{3}+\tilde{B}_{3}$, one obtains $g_{3}<0$. Since $G_{3}=g_{3}(z=0)>0$ (resulting from a numerical derivation of the curve in Fig. 3), $g_{3}$ needs to change sign along the drift. However, this also requires a change of sign in $g_{2}$ - which must be prior to the change in $g_{3}$ : From (8) it is apparent that $g_{2}=$ $\left(G_{2}-g_{1} \chi_{2}\right) / \chi_{1}^{2}$ starts with a negative value $\left(G_{2}<0\right)$, but increases, since $g_{1}>0$ while $\chi_{1}$ is negative and a function proportional to $z$. At the point $g_{2}=0$, an evaluation of $g_{3}$ yields

$$
\begin{aligned}
g_{3} & =\frac{G_{3}-g_{1} \chi_{3}-2 g_{2} \chi_{1} \chi_{2}}{\chi_{1}^{3}} \\
& =\frac{G_{3}-g_{1} \chi_{3}}{\chi_{1}^{3}}=\frac{G_{3}+G_{2} \chi_{3} / \chi_{2}}{\chi_{1}^{3}}>0,
\end{aligned}
$$

since $G_{2}$ and $\chi_{2}$ are negative while $G_{3}, \chi_{3}$ and $\chi_{1}$ are positive.

Thus, in summary: For a vanishing third order, it is required that $g_{3}$ is negative and, thus, $g_{3}$ needs to change sign in the drift between gun and buncher (since $g_{i}$ are constants in the formalism from the buncher onwards). $g_{2}$, which starts negative, can also change sign if the drift space is long enough, meaning that $g_{2}$ will reach zero at that flipping point. However, $g_{3}$ is still positive at the position where $g_{2}=0$. Since both functions $g_{2 / 3}$ should be monotone (multiple changes of the curvature and third order in phase space due to a mere drift are not physical), it is impossible that $g_{3}$ changes sign without a preceding flip in the second order curvature. Thus, there must be a minimum in the longitudinal emittance between gun and buncher cavity, indicating that behavior of the second order. This effect, which is necessary for the minimization of the third order correlation (see III B), is referred to as overcompensation mode in the subsequent paragraphs. It is depicted in Fig. 11.

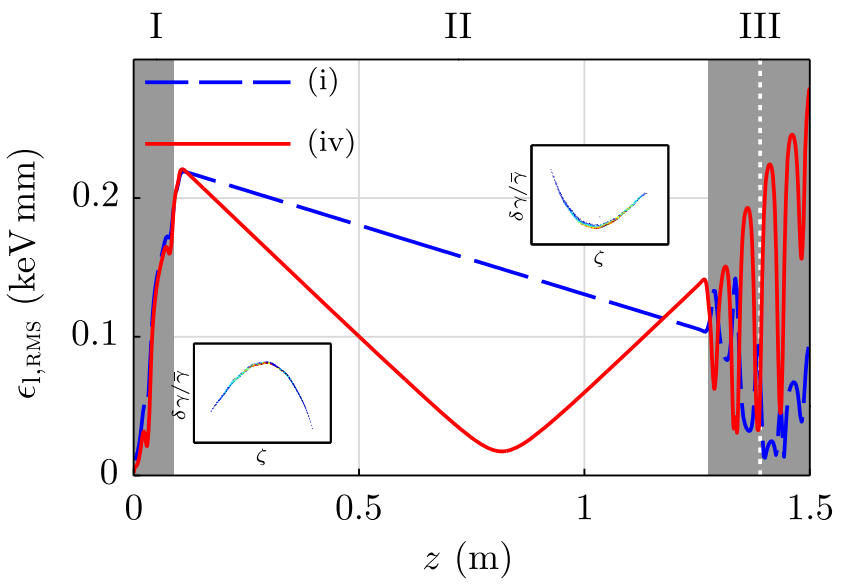

FIG. 11. Comparison of the longitudinal RMS emittance for case (i) and case (iv), resulting from AstrA simulations. For case (i), space charge was not included, and only the second order is eliminated, while case (iv) is tracked including space charge and aimed for a minimization of second and third order correlations in the focus. As can be seen, there is a minimum of the emittance in between gun and buncher cavity for case (iv), where the second order changes sign, which is illustrated by the insets showing the longitudinal phase space before and after the minimum; the linear correlation has been subtracted for demonstration purposes. Without this overcompensation mode-as in case (i) - a minimization of the third order is impossible.

\section{Energy spread compensation}

Not all experiments demand a short bunch. In some cases, a long bunch with small energy spread is required instead. For example, in the case of transmission microscope experiments [15,16], also planned at REGAE [12], the beam needs

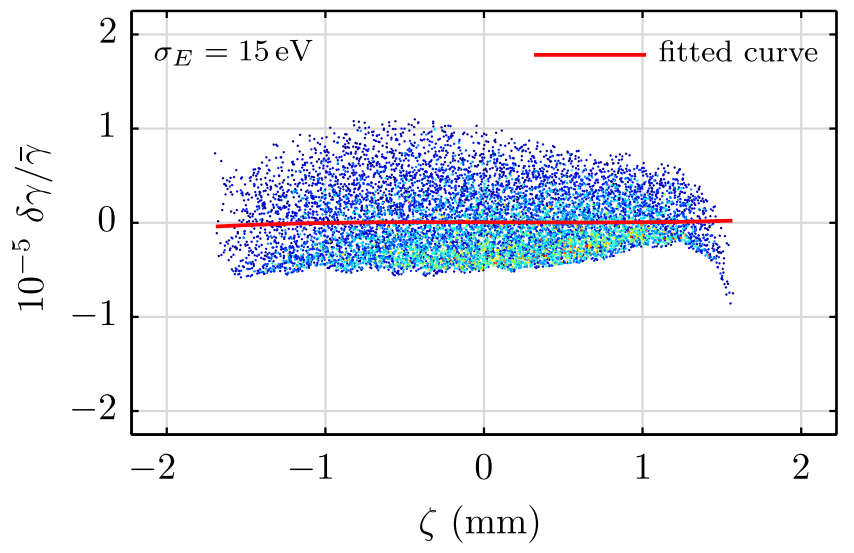

FIG. 12. ASTRA simulation for a parameter set determined by the condition $B_{i}=g_{i}+\tilde{B}_{i} \equiv 0$ for $i \in\{1,2,3\}$. The gun is set to $E_{\mathrm{G}}=100.0 \mathrm{MV} / \mathrm{m}$ and $\phi_{\mathrm{G}}=45.7 \mathrm{deg}$, while the buncher settings are $E_{\mathrm{B}}=14.1 \mathrm{MV} / \mathrm{m}$ and $\phi_{\mathrm{B}}=-69.9 \mathrm{deg}$. The resulting energy spread is about $\sigma_{\mathrm{E}_{\mathrm{kin}}}=15 \mathrm{eV}$ for a bunch energy of $E_{\text {kin }}=3.9 \mathrm{MeV}$, i.e. $\sigma_{\mathrm{E}_{\text {kin }}} / E_{\text {kin }}=4 \times 10^{-6}$. The red line results from a third order polynomial fit, clearly demonstrating that the first three orders vanish almost completely. 
to be transversely focused to a tight spot onto the target. In this case, space charge effects limit the achievable resolution in the case that a bunch is too short, while the rf induced energy spread in combination with chromatic aberrations limits the resolution when the bunch is too long. Operating the gun on a debunching phase and compensating the energy spread with the buncher cavity offers a possibility to generate long bunches with minimal energy spread. For this case, it is enough to transport the phase space up to the buncher, using Eqs. (5)-(10) and cancel the polynomial coefficients $g_{i}$ with respective coefficients $\tilde{B}_{i}$ for $i \in\{1,2,3\}$. In other words: $B_{1}=B_{2}=B_{3} \equiv 0$ from Eqs. (10) and (12).

A possible parameter set to achieve such a bunch configuration is, for example, $E_{\mathrm{G}}=100.0 \mathrm{MV} / \mathrm{m}$, $\phi_{\mathrm{G}}=48.1 \mathrm{deg}$ and $E_{\mathrm{B}}=13.5 \mathrm{MV} / \mathrm{m}, \phi_{\mathrm{B}}=-64.8 \mathrm{deg}$, according to the formalism. Using these parameters as a seed for ASTRA simulations results in a remarkably low energy spread of $\sigma_{\mathrm{E}_{\mathrm{kin}}}=15 \mathrm{eV}$, which corresponds to $\sigma_{\mathrm{E}_{\mathrm{kin}}} / E_{\mathrm{kin}}=4 \times 10^{-6}$. Space charge forces are included in this simulation with a charge of $50 \mathrm{fC}$; the cavity parameters for the gun are tuned to $E_{\mathrm{G}}=100.0 \mathrm{MV} / \mathrm{m}$ and $\phi_{\mathrm{G}}=45.7 \mathrm{deg}$, while the buncher is set to $E_{\mathrm{B}}=$ $14.1 \mathrm{MV} / \mathrm{m}$ and $\phi_{\mathrm{B}}=-69.9 \mathrm{deg}$.

In this case, it is instructive to look at the longitudinal RMS emittance once again. As can be seen from Fig. 13, there is a minimum in the emittance in between the two cavities, i.e., the machine is set to overcompensation mode, the curvature flips over and changes sign along the drift. As a consequence, the curvature compensation can be achieved on an accelerating phase in the buncher, which has the same sign in the curvature as the gun cavity. The result is a slight increase of energy to about
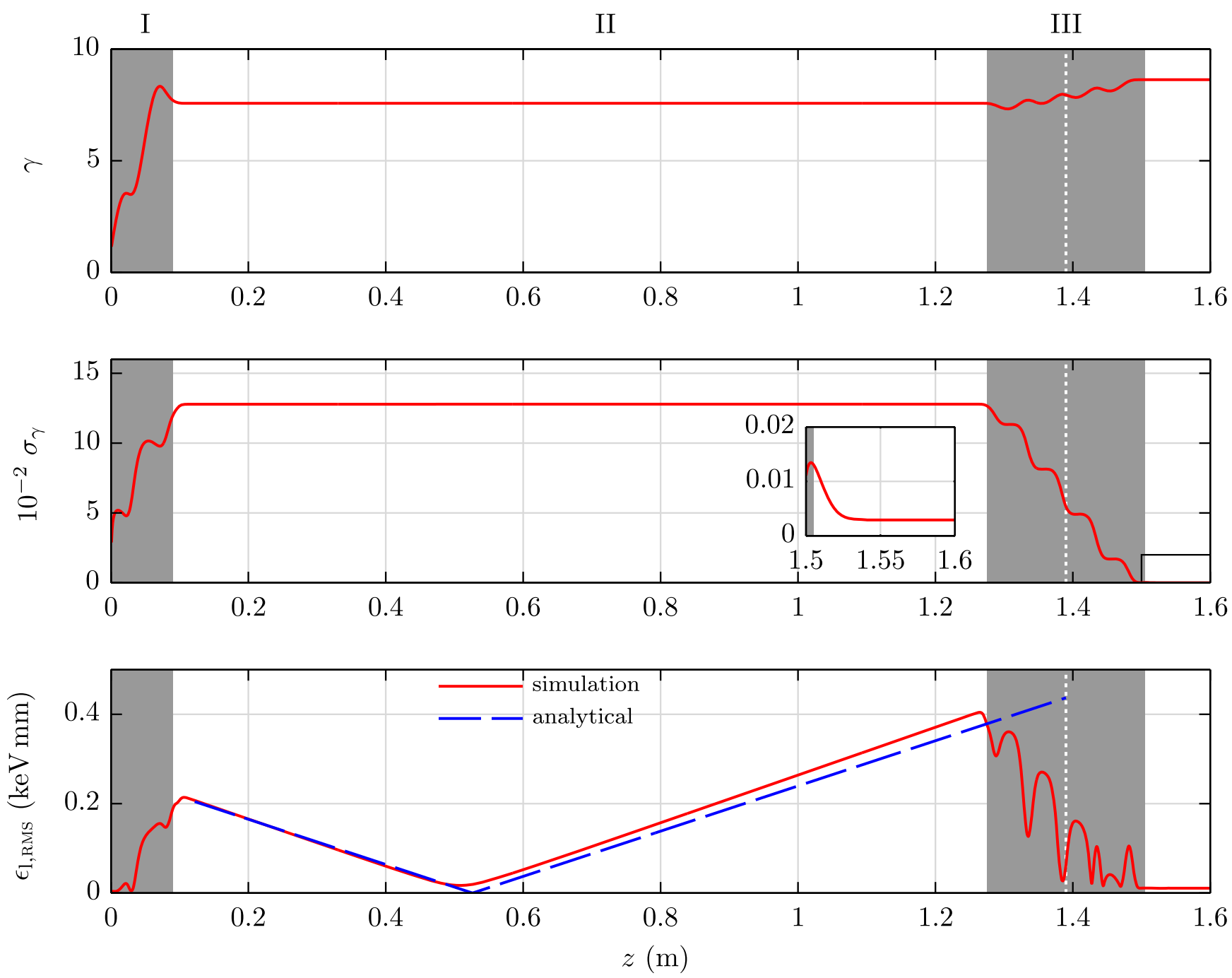

FIG. 13. Evolution of energy $\gamma$, RMS energy spread $\sigma_{\gamma}$ and longitudinal RMS emittance $\epsilon_{1, \text { RMS }}$ of a bunch for the energy spread compensation parameters discussed in the text and Fig. 12. Due to the change in sign in the second order correlation, indicated by the local minimum of the emittance between the cavities, the energy spread compensation is achieved on an accelerating phase, meaning that the overall energy, $\gamma$, is increasing in the second cavity, while $\sigma_{\gamma}$ almost vanishes. 
$E_{\text {kin }}=3.9 \mathrm{MeV}$-corresponding to $\gamma=8.6$-in contrast to the deceleration present in the bunching configurations.

Such a low energy spread will of course be hard to measure and might be polluted by uncorrelated energy deviations, for example, by the work function of the cathode. However, if the approach can be verified, it will be a very interesting topic for (ultrafast) transmission electron microscopy (UEM/TEM), which is also one of the REGAE goals: Such a low energy spread drastically reduces chromatic aberrations, enabling higher resolution. The method presented here is especially interesting as it does not require a higher harmonic cavity and the increase in energy, in contrast to energy loss accompanied by a third harmonic structure.

\section{E. Comment on the transverse dynamics}

The standard operating phase of an $\mathrm{rf}$ gun, where typically the minimal transverse emittance is achieved, is close to the phase of maximal energy gain $\left(\phi_{\mathrm{G}}=0 \mathrm{deg}\right)$. Operation at a debunching phase is quite unusual. A detailed study of the transverse dynamics in the debunching phase range, especially with significant space charge, is beyond the scope of this article. However, a few comments can be made.

Due to phase slippage of the emitted electron with respect to the accelerating field, the extraction field at the standard operation point during emission is only about $60 \%$ of the maximal gradient, because the emission phase is about $\phi_{\mathrm{em}}=40 \mathrm{deg}$, as can be seen from Fig. 14 . A higher extraction field leads in general to a smaller emittance because the spot size of the laser beam on the cathode can be reduced. At the debunching phase the emission phase is close to $\phi_{\mathrm{em}}=90$ deg, i.e., the sinusoidal extraction field is almost maximal, which thus allows

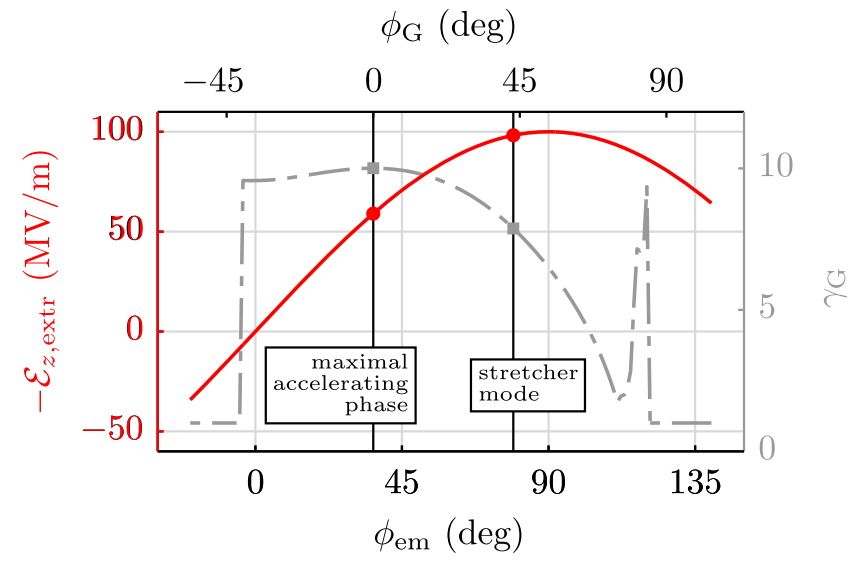

FIG. 14. Comparison of the emission phase, $\phi_{\mathrm{em}}$, and the gun phase, $\phi_{\mathrm{G}}$. There is a constant offset between these two phase definitions of about $40 \mathrm{deg}$ in this example. The extraction field (red solid line) is about $60 \mathrm{MV} / \mathrm{m}$ at $\phi_{\mathrm{G}}=0 \mathrm{deg}$ and increases towards higher gun phases. The gray dash-dotted line depicts the energy gain in the gun. to produce a beam with higher initial phase space density. Furthermore, the initial bunch length can be increased-as in the case of a setup with higher harmonic cavity-which leads to a reduction and - via the beam aspect ratio- to a linearization of the space charge forces. The large induced correlated energy spread is, however, detrimental, at least in a high bunch charge case where an emittance compensation process needs to be employed [17]. This case requires detailed studies, but it is expected that emittance compensation is less effective for a beam with large energy spread unless new concepts are found. Also, rf-induced emittance deserves attention for the long bunches envisioned here. As pointed out in [14], a chromatic emittance variation in the rf gun and the following drift can lead to an increase of the rf induced emittance at high emission phase, which needs to be controlled. An optimization of the transverse emittance for the low charge cases discussed in this article has not been attempted, but comparisons to the case where the bunch is emitted on the standard phase show only a slight degradation. Studies on further improvements are ongoing.

\section{DISCUSSION AND OUTLOOK}

In summary, in this article a method is developed and analyzed which allows for the compensation of nonlinear correlations in the longitudinal phase space of an electron bunch. The concept relies on the expansion of the bunch (stretcher mode) in order to cover an increased phase interval in a subsequent accelerating cavity-which thus acts similar to a higher harmonic structure in phase space coordinates.

The formalism based on an analytic description of the bunch evolution up to the third order agrees very well with simulations using ASTRA. It predicts the feasibility to generate bunches with a length below 1 fs for low charge without the use of a higher harmonic system. This is interesting for time-resolved electron diffraction or the external injection experiment at REGAE, where a bunch of this conventional accelerator is planned to be injected into a laser-driven plasma wakefield [13]. Alternatively, flat bunches in terms of energy spread well below $100 \mathrm{eV}$ can be produced, which could enhance the resolution of ultrafast transmission electron microscope experiments due to the reduced chromatic aberrations.

The numbers determined are only exemplary, since the focus lies on the demonstration of the approach and not on the shortest bunch possible. Tolerance studies and necessary synchronization requirements, as well as higher charge cases, have to be explored in the future work. The latter is especially interesting, since the off-crest acceleration in the gun coincides with a higher field strength at the cathode, reducing space charge influence at the extraction.

Also accompanied with the off-crest acceleration is the so-called overcompensation mode, characterized by a first minimum of the longitudinal RMS emittance before the 
bunch enters the second cavity, meaning that the second order correlation in phase space flips over and changes its sign. This enables the energy spread compensation on an accelerating phase and is also a requirement in order to eliminate the third order contribution in the longitudinal focus of a bunching configuration. Since the calculations for this article are made with the REGAE configuration in mind, the parameter space for possible solutions is limited. Allowing for a longer drift between gun and buncher could result in an overcompensation configuration at lower gun phases (and thus higher energy), and/or even in an accelerating bunching phase in the buncher cavity.

Very short bunches can be reached at a few $\mathrm{MeV}$ beam energies only at very low bunch charge, since space charge repulsion is too strong. An increase of charge and energy in a ballistic bunching scenario is conceivable by injecting the bunch before the longitudinal focus is reached into another acceleration section, in order to freeze out the longitudinal focus at higher energy. (A partly comparable method has been successfully used at the CEBAF injector, however for a very different machine configuration [18].) Furthermore, the basic concept of our approach, i.e., to stretch the bunch in order to linearize the longitudinal phase space with the fundamental rf frequency, can be applied also to magnetic bunch compression scenarios. Thus it is applicable at higher energies and higher charge, which is especially interesting for facilities based on X-band technology where higher harmonic rf systems are not readily available. Limitations of such a generalization arise from space charge in the low energy section at the start and the high energy spread imprinted at the gun which can spoil the transverse beam dynamics in that region. This is, for example, a possible scenario if a so-called emittance compensation scheme is used, which relies on a delicate transverse focusing optics. The absence of a buncher cavity is not a limitation, but requires an adaption of the mathematical formulation, since for that case the thin lens approximation breaks down.

\section{ACKNOWLEDGMENTS}

We want to thank I. Dornmair for valuable input and stimulating discussions. We also want to express our gratitude to A.C. Yannias and P. A. Walker for proof reading.

[1] Relativistic Electron Gun for Atomic Exploration REGAE, regae.desy.de.

[2] M. Hada, J. Hirscht, D. Zhang, S. Manz, K. Pichugin, D. Mazurenko, S. Bayesteh, H. Delsim-Hashemi, K. Floettmann, M. Huening, S. Lederer, G. Moriena, C. Mueller, G. Sciaini, and D. Miller, REGAE: New source for atomically resolved dynamics, in Research in
Optical Sciences (Optical Society of America, Washington, D.C., 2012) p. JT2A.47.

[3] K. Floettmann, Generation of sub-fs electron beams at few-MeV energies, Nucl. Instrum. Methods Phys. Res., Sect. A 740, 34 (2014).

[4] X. H. Lu, C. X. Tang, R. K. Li, H. To, G. Andonian, and P. Musumeci, Generation and measurement of velocity bunched ultrashort bunch of pC charge, Phys. Rev. ST Accel. Beams 18, 032802 (2015).

[5] C. R. Prokop, P. Piot, B. E. Carlsten, and M. Church, Beam dynamics performances and applications of a low-energy electron-beam magnetic bunch compressor, Nucl. Instrum. Methods Phys. Res., Sect. A 719, 17 (2013).

[6] K. Floettmann, ASTRA-A space charge tracking algorithm, http://www.desy.de/ mpyflo/.

[7] T. I. Smith, Intense low emittance beams for free electron lasers, in Proceedings of the 1986 Linear Accelerator Conference (LINAC86) (SLAC, Stanford, CA, 1986), p. 421; Report No. SLAC-R-PUB-303.

[8] K. Floettmann, T. Limberg, and P. Piot, Generation of ultrashort electron bunches by cancellation of nonlinear distortions in the longitudinal phase space, TESLA-FEL Report No. 2001-06, 2001.

[9] F. Fu, R. Wang, P. Zhu, L. Zhao, T. Jiang, C. Lu, S. Liu, L. Shi, L. Yan, H. Deng, C. Feng, Q. Gu, D. Huang, B. Liu, D. Wang, X. Wang, M. Zhang, Z. Zhao, G. Stupakov, D. Xiang, and J. Zhang, Demonstration of Nonlinear-EnergySpread Compensation in Relativistic Electron Bunches with Corrugated Structures, Phys. Rev. Lett. 114, 114801 (2015).

[10] E. Vogel, C. Albrecht, N. Baboi, C. Behrens, T. Delfs, J. Eschke, C. Gerth, M. G. Hoffmann, M. Hoffmann, M. Hüning, R. Jonas, J. Kahl, D. Kostin, G. Kreps, F. Ludwig, W. Maschmann, C. Müller, P. Nommensen, J. Rothenburg, H. Schlarb, C. Schmidt, J. Sekutowicz, H. Edwards, E. R. Harms, A. Hocker, T. N. Khabiboulline, and M. Kuhn, Test and commissioning of the third harmonic rf system for FLASH, in Proceedings of the International Particle Accelerator Conference, Kyoto, Japan (ICR, Kyoto, 2010), p. 4281, THPD003.

[11] R. Brinkmann, K. Flöttmann, J. Roßbach, P. Schmüser, N. Walker, and H. Weise, TESLA-Technical design report, Part II, Report No. DESY 2001-011, Chap. 9, p. 281.

[12] S. Manz, A. Casandruc, D. Zhang, Y. Zhong, R. A. Loch, A. Marx, T. Hasegawa, L. C. Liu, S. Bayesteh, H. DelsimHashemi, M. Hoffmann, M. Felber, M. Hachmann, F. Mayet, J. Hirscht, S. Keskin, M. Hada, S. W. Epp, K. Flöttmann, and R. J.D. Miller, Mapping atomic motions with ultrabright electrons: towards fundamental limits in space-time resolution, Faraday Discuss. 177, 467 (2015).

[13] B. Zeitler, I. Dornmair, T. Gehrke, M. Titberidze, A. R. Maier, B. Hidding, K. Flöttmann, and F. Grüner, Merging conventional and laser wakefield accelerators, in SPIE Optics + Optoelectronics, Vol. 8779, edited by E. Esarey, C. B. Schroeder, W. P. Leemans, K. W. D. Ledingham, and D. A. Jaroszynski (Society of Photographic Instrumentation Engineers, Bellingham, Washington, 2013), p. 877904.

[14] K. Floettmann, rf-induced beam dynamics in rf guns and accelerating cavities, Phys. Rev. ST Accel. Beams 18, 064801 (2015). 
[15] R. K. Li and P. Musumeci, Single-Shot MeV Transmission Electron Microscopy with Picosecond Temporal Resolution, Phys. Rev. Applied 2, 024003 (2014).

[16] D. Xiang, F. Fu, J. Zhang, X. Huang, L. Wang, X. Wang, and W. Wan, Accelerator-based single-shot ultrafast transmission electron microscope with picosecond temporal resolution and nanometer spatial resolution, Nucl. Instrum. Methods Phys. Res., Sect. A 759, 74 (2014).
[17] L. Serafini and J. B. Rosenzweig, Envelope analysis of intense relativistic quasilaminar beams in rf photoinjectors: A theory of emittance compensation, Phys. Rev. E 55, 7565 (1997).

[18] G. A. Krafft, Correcting M56 and T566 to obtain very short bunches at CEBAF, AIP Conf. Proc. 367, 46 (1996). 